\title{
ENERGY DECAYS LOCALLY EVEN IF TOTAL ENERGY GROWS ALGEBRAICALLY WITH TIME
}

\author{
BY CLIFFORD O. BLOOM AND NICHOLAS D. KAZARINOFF \\ Communicated by Cathleen Morawetz, February 13, 1973
}

0 . Introduction. In this note we announce energy decays locally like $t^{-2+\kappa}$ for solutions of hyperbolic equations, with coefficients that depend upon both position and time, in the exterior of star-shaped domains in $\boldsymbol{R}^{3}$. Here $\kappa$ is a positive constant, depending on the coefficients, defined explicitly by (8) below. Our results generalize those of Zachmanoglou [4]. $\mathrm{He}$ considered a class of equations with time-independent coefficients (see (12) below) and proved under hypotheses roughly analogous to ours that in $\boldsymbol{R}^{n}(n \geqq 3)$ energy decays locally like $t^{-1+\mu}(1>\mu \geqq 0)$. A more important difference between the equations we consider here and those considered by Zachmanoglou in [4] is that we treat equations with solutions whose total energy may grow algebraically with $t$ while the total energy of solutions of the equations considered in [4] is conserved. In [1] we proved that the energy of solutions with bounded total energy decays locally like $t^{-2}$, but under more stringent hypotheses than those used here.

We now set the scattering problem whose solutions we investigate. Let $V$ be the exterior of a closed, bounded subset $B$ of $\boldsymbol{R}^{3}$, and let $n$ be the outward unit normal to $\partial B$. We assume that the origin lies interior to $B$ and that $\partial V \equiv \partial B$ is star-shaped:

$$
\min _{x \in \partial V} \frac{n \cdot x}{r} \geqq 0,
$$

where $x=\left(x_{1}, x_{2}, x_{3}\right)$ and $r^{2}=x \cdot x$. Let $Q=(V \cup \partial V) \times[0, \infty)$. We use the notation $\nabla^{(3)}=\left(\partial / \partial x_{1}, \partial / \partial x_{2}, \partial / \partial x_{3}\right), \nabla=\left(\nabla^{(3)}, \partial / \partial t\right)$. We take as given a symmetric $3 \times 3$ matrix $E, 1 \times 3$ matrices $a$ and $b$, and functions $c$ and $d$ which satisfy the following hypothesis:

$\left(\right.$ Hypothesis $\mathrm{H}_{1}$ ) (a) $b$, c and $E$ are in $C^{1}(Q) ; a$ and $d$ are in $C^{2}(Q)$, (b) for some $d_{0}>0, d(x, t) \geqq d_{0}$ if $(x, t) \in Q$.

Let the transpose of a matrix $M$ (or $m$ ) be $M^{T}$ (or $m^{T}$ ). We suppose that $E$ is uniformly elliptic in $Q$, namely that there exist positive constants $c_{0}$ and

AMS (MOS) subject classifications (1970). Primary 35B05, 35L10. 78A45; Secondary 35P25, 78A05.

Key words and phrases. Energy decay, star-shaped obstacle, second order hyperbolic equations, variable coefficients, divergence identity. 
$C_{0}$ such that

$$
C_{0} \geqq \max _{|\xi|=1} \xi E \xi^{T} \geqq \min _{|\xi|=1} \xi E \xi^{T} \geqq c_{0} .
$$

Finally we adopt the notation

$$
A=\left(\begin{array}{cc}
E & a^{T} \\
a & -d
\end{array}\right), \quad D=\left(\begin{array}{cc}
E_{t} & b^{T} \\
b & -c
\end{array}\right)
$$

and

$$
(\hat{\bullet})=\frac{(\cdot)}{\min _{|\dot{\xi}|=1} \xi E \xi^{T}} .
$$

We consider solutions of the mixed initial boundary value problem

$$
\begin{gathered}
\nabla\left(A(\nabla u)^{T}\right)+\left(b-a_{t}\right) \cdot \nabla^{(3)} u+\frac{1}{2}\left(d_{t}-c\right) u_{t}=0 \quad(x \in V, t>0), \\
u(x, t)=0 \quad(x \in \partial V, t \geqq 0), \\
u(x, 0)=f(x), u_{t}(x, 0)=g(x) \quad(x \in V),
\end{gathered}
$$

where $f$ and $g$ are functions in $C^{1}(V \cup \partial V)$ with compact support.

1. Norms and constants. We use the following norms:

$$
N(\cdot) \equiv \max _{V \cup \partial V}|\cdot| \text { and } N^{\prime}(\cdot) \equiv \max _{t \geqq 0} N(\cdot)
$$

We shall assume that the coefficients $c, d$ and the matrix coefficients $a, b$, and $E$ are such that the following are positive numbers:

$$
\begin{aligned}
\alpha_{1}= & 2 N^{\prime}\left(r d_{r} / d\right)+2 N^{\prime}\left(r a_{r} / d\right)+N^{\prime}\left(r\left(d_{t}-c\right) / d\right), \\
\alpha_{2}= & 2 N^{\prime}\left(r \hat{E}_{r}\right)+2 N^{\prime}\left(r \hat{a}_{r}\right)+4 N^{\prime}\left(r\left(\hat{a}_{t}-\hat{b}\right)\right)+N^{\prime}\left(r\left(\hat{d}_{t}-\hat{c}\right)\right) \\
& +8 N\left(r^{-2}\right) N^{\prime}\left[r^{4}\left(\frac{1}{2}\left(d_{t}-c\right)_{t}-\nabla^{(3)} \cdot\left(a_{t}-b\right)\right)\right] N^{\prime}(\hat{1}), \\
\alpha_{1}^{\prime}= & N^{\prime}(t b / d)+N^{\prime}(t c / d), \quad \alpha_{2}^{\prime}=N^{\prime}\left(t \hat{E}_{t}\right)+N^{\prime}(t \hat{b}) .
\end{aligned}
$$

In addition to the smoothness conditions on the coefficients already imposed, this hypothesis imposes decay rates on the coefficients in (1) and on their time and space derivatives.

Let $\Omega$ be defined by the equation

$$
\begin{aligned}
&\left\{1+N^{\prime}(\hat{d})+N^{\prime}\left(r^{-2}\right)\right. N^{\prime}(\hat{1})\left[4 N^{\prime}\left(r^{3} d_{r}\right)+2 N^{\prime}\left(r^{3}\left(d_{t}-c\right)\right)\right] \\
&\left.+4\left(N^{\prime}\left(r^{-4}\right)\right)^{1 / 2} N^{\prime}(\hat{1}) N^{\prime}\left(r^{2} a\right)+2 N^{\prime}(\hat{a})\right\} \Omega=\frac{1}{2} .
\end{aligned}
$$

Again we shall assume that the coefficients of (1) are such that the above norms are well defined.

For any $\varepsilon \in(0,1)$, each $T>0$, and some small $\delta>0$, we define

$$
\begin{gathered}
\mathscr{D}(T)=\{x \mid r \leqq \varepsilon \Omega T\} \cap V, \\
\kappa=(1-\varepsilon)^{-1}\left[\max \left(\alpha_{1}, \alpha_{2}\right)+\left(1+(\varepsilon \Omega)^{2}\right) \max \left(\alpha_{1}^{\prime}, \alpha_{2}^{\prime}\right)\right]
\end{gathered}
$$




$$
s=\max \left[N^{\prime}\left(t \hat{E}_{t}\right)+N^{\prime}(t \hat{b}), N^{\prime}(t b / d)+N^{\prime}(t c / d)\right]
$$

and

$$
q=-1+\delta+s
$$

Note that if the differential equation (2) is the wave equation, then $\kappa=0$.

2. Local energy decay. We make the following major hypothesis:

$\left(\right.$ Hypotheses $\left.\mathrm{H}_{2}\right)$ The $N^{\prime}$-norm of each of the following is a positive number:

$$
\begin{aligned}
& r^{3+q}(E-d I), \quad r^{4+q} E_{r}, \quad r^{4+q} d_{r}, \quad r^{4+q}\left(d_{t}-c\right), \quad r^{3+q} a, \\
& r^{4+q} a_{r}, \quad r^{4+q}\left(a_{t}-b\right), \quad r^{4+q}\left[2\left(d_{t}-c\right)+d_{t}-2 \nabla^{(3)} \cdot a\right], \\
& r^{5+q}\left(d_{t}-c\right)_{t}-2 \nabla^{(3)} \cdot\left(a_{t}-b\right), \quad t E_{t}, r^{4+q} E_{t}, t b, \quad \text { and } t^{4+q} c .
\end{aligned}
$$

Let $\mathscr{E}_{\text {loc }}(x, t)=\frac{1}{2} \int_{\mathscr{D}}\left[d u_{t}^{2}+\nabla^{(3)} u E\left(\nabla^{(3)} u\right)^{T}\right] d x$, and let $\mathscr{E}(x, 0)$ be the total initial energy associated with $u$.

THEOREM 1. Suppose that $\partial V$ is star-shaped, E is uniformly strongly elliptic, Hypothesis $\mathrm{H}_{1}$ is satisfied and the initial data $(f, g)$ in (4) are smooth and have compact support in $V$. Then the unique solution to Problems (2)-(4) has compact support. Moreover, suppose $T_{0} \equiv T_{0}(\varepsilon)$ is so large that $\partial V \subset \mathscr{D}\left(T_{0}\right)$, where $\mathscr{D}$ is defined by (7) and Hypothesis $\mathrm{H}_{2}$ is satisfied by the coefficients of the differential equation (2). Then, for each $\varepsilon \in(0,1)$, there exist positive constants $M, K$, and $\kappa$, with $\kappa$ defined by (8), such that for $T>T_{0}$

$$
\mathscr{E}_{1 \mathrm{oc}}(x, T) \leqq \frac{K \mathscr{E}(x, 0)}{T^{2}}\left\{1+\left(\frac{T}{T_{0}}\right)^{\kappa} e^{M / T_{0}}\left[1-\left(\frac{T_{0}}{T}\right)^{\kappa}+\frac{M}{(1+\kappa) T_{0}}\right]\right\}
$$

The theorem holds even if for each positive number $p$ there exists a $t>p$ for which the quadratic form associated with the matrix $D$ defined by (1) fails to be negative semidefinite on $V \cup \partial V$. The negative semidefiniteness of $D$ was a major hypothesis in [1].

COROLlaRY 1. If the quadratic form associated with $D$ is negative semidefinite on $(V \cup \partial V) \times\left[T_{0}, \infty\right)$, then the energy decay estimate (11) holds with $\alpha_{1}^{\prime}$ and $\alpha_{2}^{\prime}$, which enter into the definition of $\kappa$, both zero and with the exponent $q$ in Hypothesis $\mathrm{H}_{2}$ equal to $-1+\delta$.

In the case of the wave equation our decay estimate reduces to $\mathcal{O}\left(t^{-2}\right)$, which is the same as that obtained by C. S. Morawetz in [2]. E. C. Zachmanoglou [4] proved energy decays locally like $t^{-1+\mu}(1>\mu \geqq 0)$ for solutions of hyperbolic equations of the form

$$
\nabla^{(3)}\left(E(x)\left(\nabla^{(3)} u\right)^{T}\right)-c(x) u-d(x) u_{t t}=0
$$


by generalizing the argument used by Morawetz in [3] in treating the wave equation. To establish a faster rate of energy decay as $t \rightarrow \infty$ than the rate Zachmanoglou establishes in [4], we have to impose Hypothesis $\mathrm{H}_{2}$, which is more stringent by a factor of $r$ than his analogous conditions. But the total energy of solutions of equations of the form (2) satisfying Hypothesis $\mathrm{H}_{2}$ may grow algebraically with $t$. In [1] we considered equation (2) with $E=E(x, t), c=0$, and $d=1$ under the hypotheses that $E_{t} \leqq 0, c_{0} \geqq 1$, and for $t \geqq N$ and $r \geqq \varepsilon t+c,\left|r \nabla^{(3)} E\right|=\mathcal{O}\left(t^{-2-\delta}\right)$, $|x(E-I)|=\mathcal{O}\left(t^{-1-\delta}\right)$, and $\left|r E_{r}\right|=\mathcal{O}\left(t^{-2-\delta}\right)$ for some positive $c$ and $\delta$.

Our methods of proof in [1] and of Theorem 1 are similar; the estimates we use to prove Theorem 1 are much sharper. Both results are based on the divergence identity

$$
\begin{aligned}
& \nabla\left[(\alpha \cdot \nabla u)(A w)-\alpha^{T}(\nabla u A w) / 2+u(\gamma A+B) w+C^{T} u^{2} / 2\right] \\
& \equiv[\alpha \cdot \nabla u+\gamma u](\nabla(A w))+(\nabla \cdot C) u^{2} / 2+u[C+(\nabla \gamma) A+\nabla \cdot B] \cdot \nabla u \\
& \quad+\nabla u[\gamma A-(\nabla \cdot \alpha) A / 2-(\alpha \cdot \nabla) A / 2+\nabla \alpha A] w,
\end{aligned}
$$

where $B$ is an antisymmetric $4 \times 4$ matrix with $B^{i *}=0(i=1,2,3)$, $w=(\nabla u)^{T}, B^{4 *}=\left[-\left(\beta+t^{2} r^{-2}\right) x, 0\right] d, \alpha=\left(2 x t, r^{2}+t^{2}\right), \gamma=2 t, C=$ $\left(2 t x d r^{-2}+\Delta d,-d-t^{2} d r^{-2}\right), \beta$ is a solution of

$$
(d \beta)_{r}+3(d \beta) r^{-1}=3 d r^{-1}+t\left(d_{t}-c\right) r^{-1}+t^{2} d_{r} r^{-2},
$$

$\nabla \alpha=\left(\alpha_{x_{i}}^{j}\right)$, and $\Delta d=2 t\left(b-a_{t}\right)-2 a+\left[(\beta d)_{t}+\left(t^{2} d\right)_{t} r^{-2}\right] x-2 t x d r^{-2}$. We integrate this identity over the space-time domain bounded by the planes $t=T_{0}, t=T\left(T>T_{0}\right)$, and the surface $\partial V \times\left[T_{0}, T\right]$, apply the divergence theorem, and estimate carefully to prove Theorem 1 .

\section{REFERENCES}

1. C. O. Bloom and N. D. Kazarinoff, Energy decay in nonhomogeneous media, Proc. Sympos. on Analysis (Rio de Janeiro August 15-24, 1972), Hermann, Paris (to appear).

2. Cathleen S. Morawetz, The limiting amplitude principle, Comm. Pure Appl. Math. 15 (1962), 349-361. MR 27 \#1696.

3. - The decay of solutions of the exterior initial-boundary value problem for the wave equation, Comm. Pure Appl. Math. 14 (1961), 561-568. MR 24 \# A2744.

4. E. C. Zachmanoglou, The decay of solutions of the initial-boundary value problem for hyperbolic equations, J. Math. Anal. Appl. 13 (1966), 504-515. MR 32 \#6055.

Department of Mathematics, Wayne State University, Detroit, Michigan 48202

Department of Mathematics, State University of New York at Buffalo, Amherst, NEW YORK 14226 